\title{
IMPACT ÉCONOMIQUE RÉGIONAL D'UN PÔLE UNIVERSITAIRE : APPLICATION AU CAS STRASBOURGEOIS
}

\author{
Laurent Gagnol * \\ Chercheur Post-doctorant \\ Jean-Alain Héraud * \\ Professeur de Sciences Économiques à l'Université Louis Pasteur \\ * Bureau d'Économie Théorique et Appliquée \\ Université Louis Pasteur, CNRS \\ P.E.G.E., 61 avenue de la Forêt Noire \\ 67085 Strasbourg Cedex
}

\begin{abstract}
Résumé
Cet article analyse les résultats d'une étude de l'impact économique d'un pôle universitaire sur son territoire local. L'université est, en effet, une structure consommatrice directe de biens et services, pouvant induire un impact significatif sur l'économie locale. Son impact direct concerne également la localisation d'un certain nombre de salariés et d'étudiants, euxmêmes acteurs économiques à part entière. Enfin, de par sa vocation d'enseignement et de recherche, l'Université agit comme un moteur du développement local, puisqu'elle pourvoit à l'amélioration du «capital humain » et agit ainsi comme une force attractive pour toute une catégorie d'entreprises à la recherche de compétences universitaires. Outre les résultats obtenus (représentatifs d'un grand site universitaire régional), cet article évoque les questions méthodologiques liées à ce type d'évaluation économique.
\end{abstract}

Mots clé : Impact économique, université, territoire, évaluation

Key-words : Economic impact, university, territory, evaluation 


\section{INTRODUCTION}

L'objectif de cet article est de présenter les principaux résultats d'une analyse concernant les universités de Strasbourg (avec les écoles et les organismes de recherche associés), visant à améliorer la connaissance de l'impact économique d'un pôle d'enseignement supérieur et de recherche sur son environnement local. Peu d'études de ce type avaient été menées jusqu'à présent, en tout cas en Europe, bien que le besoin s'en fasse sentir de plus en plus. Cette étude d'impact intéresse les collectivités de différents niveaux qui contribuent au financement du complexe universitaire (en particulier à travers les contrats de Plan État-Région), les établissements eux-mêmes et divers acteurs qui interviennent dans le processus d'investissement public (1).

Pour les collectivités territoriales, qui consacrent désormais des sommes importantes à l'enseignement et à la recherche, les retombées économiques des investissements publics méritent d'être précisément définies, comme le souligne A. Sauvage (1994). Pour les universités elles-mêmes, une étude de leur positionnement régional peut permettre de révéler les points forts mais également de souligner les faiblesses de l'activité académique, et de construire des argumentaires vis-à-vis des partenaires économiques et sociaux, à une époque où cette activité est de plus en plus tenue de se légitimer (2).

Pour mener à bien une telle analyse, nous considérons les deux grandes fonctions de l'Université :

- consommateur, employeur et localisateur de dépenses, d'une part;

- agent économique local qui pourvoit à l'amélioration du «capital humain », à l'attraction et à la création d'entreprises via les transferts de compétences, d'autre part.

L'impact de ces fonctions théoriques a fait l'objet d'une vérification empirique, que nous avons voulu aussi objective (et méthodologiquement fondée) que possible.

Notre champ d'analyse porte sur le pôle universitaire de Strasbourg dans sa globalité, c'est-à-dire ses trois universités, respectivement à dominante scientifique, littéraire et juridique, ainsi que ses laboratoires associés du CNRS et de l'INSERM. L'aire géographique d'impact que nous avons retenue se limite au département du Bas-Rhin. En effet, celui-ci est assez rapidement apparu, au cours de l'étude, comme le territoire le plus naturel à considérer pour l'analyse d'impact : la région Alsace est un espace trop grand (à moins d'inclure aussi l'université de Haute Alsace et ses implantations de Mulhouse et de Colmar), et la Communauté Urbaine de Strasbourg un espace trop restreint (l'Université Louis Pasteur a une implantation à Haguenau et un nombre important de salariés habitant hors de la CUS).

Bien que les spécificités du territoire d'analyse se prêtent parfaitement à notre problématique, les résultats présentés dans cet article n'ont pas une portée tout à fait générale. Ils ne sont sans doute applicables qu'à des pôles universitaires français de taille comparable. L'exposé de notre méthodologie devrait être par contre utile dans tous les cas.

La littérature empirique sur l'impact économique régional de la formation et de la recherche reste relativement modeste, en dehors de l'Amérique du Nord (dont le système universitaire est relativement différent de celui d'un pays comme la France). Notre travail peut, par conséquent, constituer un apport significatif à la réflexion tant théorique qu'empirique dans ce domaine de recherche. Nous nous sommes cependant appuyés sur quelques travaux issus de la recherche internationale. La contribution majeure exploitable a été celle de F. Martin (1996) qui porte sur les retombées économiques des activités de recherche de l'Université de Montréal. L'auteur conclut sur le rôle irremplaçable de la recherche universitaire comme moteur de l'économie tant au niveau de sa consommation brute (étudiants et personnel inclus) que de sa contribution à l'amélioration du capital humain. Des résultats similaires ont été soulignés dans le travail de Baslé-Le Boulch (1999) (3), dans une étude d'impact régionale portant sur le site économique de la ville de Rennes. Les auteurs parviennent à montrer l'impact majeur de l'enseignement supérieur et de la recherche sur le développement économique 
rennais. La taille importante de l'université de Rennes, tant en termes de personnel que d'étudiants, permet en effet de créer ou d'induire plus de 19000 emplois. Cependant, si l'on en juge les résultats de F. Merrien (1994), l'impact de l'université sur le développement économique local reste très difficile à cerner avec précision et demeure en tout cas fortement dépendant de la structure et de la taille des entreprises locales.

Cet article présente, dans une première section, le positionnement méthodologique et théorique de notre travail et insiste sur les rôles attendus d'une infrastructure universitaire dans son environnement local. La seconde section s'intéresse essentiellement à l'impact économique direct des universités. Il s'agit alors d'estimer les retombées des consommations des établissements d'enseignement et de recherche en se focalisant sur les dépenses d'équipement et de fonctionnement. La troisième section insiste sur l'impact direct des consommations du personnel enseignant et administratif et des étudiants. Cette approche privilégie ainsi la fonction de localisation d'une population importante sur le territoire local. Nous montrons que cet impact s'avère nettement significatif compte tenu des montants de salaires et de dépenses potentielles induites dans l'économie alsacienne. La quatrième section a pour objectif de quantifier, avec les précautions d'usage, l'impact direct en termes d'emplois du pôle universitaire. La cinquième section de notre travail s'attache à définir l'impact indirect des universités à travers leurs fonctions d'éducation, de formation de la main d' auvre, et le développement des synergies de recherche et de développement avec les entreprises régionales (transferts de compétences et localisation d'entreprises).

\section{- I -}

\section{POSITIONNEMENT THÉORIQUE ET MÉTHODOLOGIQUE}

Le domaine auquel se rattache notre travail est celui de l'évaluation des infrastructures publiques. L'évaluation est une démarche plus complexe que l'étude d'impact, puisqu'il s'agit de rapporter un résultat (un impact) à un objectif initial et aux moyens déployés. C'est aussi une démarche plus qualitative, qui intègre diverses formes de mesures (elles-mêmes quantitatives ou qualitatives) en un tout qui est supposé apporter plus que la somme de ses parties. L'expérience dont nous rendons compte ici est, plus modestement, une étude d'impact économique. Mais en présentant un ensemble de mesures quantitatives relatives au pôle universitaire strasbourgeois nous contribuons à former une vision de l'université qui ne saurait être neutre. Il n'est pas concevable de mener une étude d'impact et de rendre compte de ses résultats sans se référer au contexte général du sujet (ici le système universitaire et les fonctions qu'il remplit en principe) et exprimer une certaine approche par les choix méthodologiques qui sont faits. En un mot: l'étude d'impact n'est jamais nai ve, elle présuppose une forme d'évaluation.

Quels sont donc, théoriquement, les objectifs d'une infrastructure publique comme un pôle universitaire régional du type de Strasbourg, comportant des établissements d'enseignement supérieur et de recherche, facultés et écoles d'ingénieurs, ainsi que des organismes de recherche associés? Et quelles sont les modalités locales/régionales des fonctions remplies? On peut se référer, par exemple, au concept d'ITI (Institution of technological infrastructure) développé pour caractériser les capacités innovatrices des régions (A. Bureth, J-A. Héraud, 2001). Un ITI peut remplir trois grands types de fonctions, en termes cognitifs: (a) l'enrichissement et la gestion de la base commune de connaissances; (b) l'interface, voire la mise en réseau, des acteurs de l'innovation; (c) le conseil ou l'expertise qui font évoluer les compétences des acteurs pris individuellement. Les universités et centres de recherche contribuent essentiellement à produire de la science et à la diffuser (a); ainsi qu'à former et (plus marginalement) conseiller (c). Les fonctions d'interface (b) sont beaucoup moins du ressort du monde académique, même si divers mécanismes incitatifs l'y poussent via des opérations de transfert de technologie, de création d'entreprises, etc. (voir en particulier, en France, la loi sur l'innovation de juillet 1999). 
L'impact économique d'une université peut s'observer sur ces divers champs. Les mesures directes d'impact sur les fonctions de base sont beaucoup moins fréquentes en France (et plus généralement en Europe) qu'en Amérique du Nord. En effet, si l'on prend une fonction de type (a) comme l'éducation, comme il ne s'agit pas en France d'un bien privé, on ne dispose pas d'une évaluation par le marché de cette contribution (4). De même, la recherche académique est mesurable par son coût (en équipement, fonctionnement et salaires), mais pas par une quelconque valeur de marché à l'exception de quelques aspects: la part de connaissance appliquée qui est protégée par des brevets (faible en France, alors qu'elle est significative dans une grande université scientifique américaine) est mesurable par le flux des royalties actualisées qu'elle génère. D'une manière générale, les travaux théoriques et empiriques s'intéressent plutôt aux impacts dynamiques et souvent indirects comme les potentialités de transfert de technologie, de création de "jeunes pousses", l'attractivité accrue du territoire (concrète ou par effet d'image), etc. Il faut dire que ces impacts correspondent à des objectifs politiques des acteurs nationaux et régionaux, comme on peut en voir citer en abondance typiquement dans les Contrats de Plan État-Région (voir DATAR, 2000). Mais le point de vue que nous défendons ici est que l'on manque de données sur les fonctions les plus élémentaires de l'université, comme le fait d'attirer et de fixer une population d'étudiants sur une zone géographique assez réduite.

La question théorique et méthodologique qui se pose ensuite est celle du calcul d'impact indirect ou induit. En effet, pour ne prendre que l'exemple de la population étudiante, à laquelle nous verrons qu'on peut associer l'essentiel de l'impact économique local direct, il convient d'ajouter aux effets de premier rang une cascade de conséquences que l'on a l'habitude de condenser dans les modèles sous la forme d'un "effet multiplicateur". Cette question pose à notre avis de redoutables problèmes méthodologiques, pour ne pas dire conceptuels. C'est un des points que nous voulons souligner dans cet article. Pour illustrer notre propos, dans le cas de l'évaluation de l'Université de Californie à Irvine (voir UCI.news sur le site de l'Université), il est noté que chaque dollar dépensé dans le comté d'Orange génère 3,70 dollars dans l'économie locale. Parallèlement, chaque dollar de revenu salarial perçu localement entraînerait un surcroît d'activité économique de 2,08 dollars pour le comté. Pour légitimer cette affirmation, il est fait référence à un modèle d'économie régionale créée par la Southern California Association of Government (SCAG). Il n'est pas dans notre intention de remettre en doute la qualité scientifique de ce dernier travail. Des études d'impact réalisées en France utilisent de la même manière des multiplicateurs établis par exemple par les succursales régionales de la Banque de France. Le problème est le suivant: le multiplicateur est caractéristique du modèle construit, lequel répond à des objectifs précis et à un contexte qui ne sont pas nécessairement ceux de l'exercice d'évaluation. Pour être rigoureux, il faudrait modéliser toute l'économie régionale pour chaque opération d'évaluation. Il faut bien prendre conscience du fait que, au moins dans un pays comme la France, il n'existe plus vraiment de modélisation macroéconomique régionale et que cette dernière est rendue presque impossible par l'absence de connaissance des flux interrégionaux (une circonstance particulièrement grave pour décrire des systèmes qui sont de nos jours extrêmement ouverts).

Une solution éventuelle à ce problème a été proposée, en ayant recours à la théorie de la base économique. Pour utiliser un modèle de ce type (voir par exemple R.E. Klostermann, 1990, chap. 913) il faut pouvoir faire l'hypothèse d'un modèle causal séparant très clairement un secteur de base qui fonctionne en relation avec des marchés extérieurs et le reste de l'économie régionale qui dépend de ce secteur. On en déduit alors un multiplicateur d'emploi simple à interpréter du type: «pour un emploi basic créé par Boing dans l'aire urbaine, 0,9 emplois sont indirectement générés dans les branches non-basiques ». La difficulté, concernant l'aire urbaine strasbourgeoise, est qu'il nous paraît très irréaliste de construire une telle représentation à propos du pôle universitaire. 


\section{L'IMPACT DIRECT DES UNIVERSITÉS SUR L'ÉCONOMIE LOCALE}

L'impact direct des universités strasbourgeoises sur l'économie locale repose, d'une part, sur la fonction de consommation des établissements d'enseignement et de recherche, et d'autre part, sur leur capacité à attirer et à fixer une population d'enseignants, de chercheurs, de personnel administratif et d'étudiants. Cet ensemble de consommations, d'une ampleur significative, est un premier indicateur de l'impact économique direct sur l'économie locale. On a pu ensuite extrapoler un effet induit en termes d'emplois. L'année comptable retenue pour l'évaluation est 1996.

\section{A - Méthodologie}

Les dépenses des Universités se décomposent en deux catégories principales : les dépenses de fonctionnement (achats de petit matériel, fournitures diverses...) et les dépenses d'investissement. Ces montants, aisément disponibles, sont exploitables à condition de mettre en place une grille homogène de dépenses qui permette l'agrégation homogène des types de consommation. Il s'avère, en effet, essentiel de pouvoir dégager les secteurs économiques et industriels principalement intéressés par la présence universitaire.

La base de l'étude a consisté en une analyse précise des documents comptables avec le repérage des divers fournisseurs et leur localisation. Compte tenu des diverses entités constituant le pôle universitaire de Strasbourg (trois universités, les laboratoires associés au CNRS et à l'INSERM), certaines précautions méthodologiques ont dû être prises.

Le problème majeur a consisté, pour les quatre comptabilités simplifiées qui nous ont été transmises par les établissements, à établir des correspondances entre des rubriques comptables d'une part et des codes NAF de secteurs d'activité d'autre part. En effet, les comptabilités enregistrent les dépenses par nature mais selon une nomenclature comptable sans rapport direct avec la nomenclature des produits (NAF), laquelle, seule, permet de traduire ces dépenses en impact sur les différentes branches d'activités économiques. Aucune table de conversion de ce type n'existant, les correspondances ont été établies sur la base des intitulés de comptes, et le cas échéant, de vérifications sur la nature des achats passés sous telle ou telle rubrique auprès des agents comptables des différentes universités. Cette conversion s'est donc heurtée à différents types de problèmes :

\section{- Imprécision de certaines rubriques comptables, par rapport au niveau nécessaire à une transcription fidèle en code NAF.}

Par exemple, les rubriques "Matériel de traitement" incluent aussi bien du matériel informatique, électronique, que d'optique, ce qui correspond à des secteurs d'activité bien différenciés dans la classification INSEE. De même, les "Fournitures d'entretien et de petit équipement" comprennent aussi bien des achats de papier que de tubes à essais !

\section{- Absence possible de cohérence entre les comptabilités de chacune des universités.}

Les codes comptables ne sont pas nécessairement utilisés de la même manière selon les universités (les différences sont encore plus sensibles entre les trois universités d'une part et le CNRS d'autre part). Du fait de ces "pratiques comptables" distinctes, un même achat peut être enregistré sous deux codes comptables différents selon l'établissement.

Pour résoudre ces difficultés, des hypothèses ont dû être formulées. Elles ont consisté pour l'essentiel à choisir des clés de répartition « forfaitaires » entre différents secteurs d'activité pour certains montants inscrits sous un code comptable unique, mais correspondant à des achats diversifiés de fournitures. Le choix d'un niveau d'analyse relativement agrégé nous a également permis de pallier une partie de ces difficultés. 


\section{B - Principaux résultats}

Les dépenses des universités, du CNRS et de l'INSERM se répartissent en dépenses d'investissement et en dépenses de fonctionnement. La première catégorie comprend aussi bien des dépenses en matériels de bureau, informatique, de laboratoire qu'en construction de bâtiments neufs. Sur le dernier point, on s'est résolu à exclure de notre étude des dépenses dont l'amortissement intervenait sur du long terme et dont les montants variables pouvaient biaiser significativement les résultats de notre étude portant sur la seule année 1996.

Les dépenses de construction auraient aisément pu être intégrées à l'analyse, à condition de travailler sur un horizon temporel suffisamment long pour préserver la rigueur méthodologique nécessaire. Dans nos analyses d'impact plus agrégées, nous les réintroduisons sous la forme d'une estimation annuelle moyenne (100 MF, montant essentiellement financé dans le Contrat de Plan État-Région et non sur budget propre des établissements).

\section{a) Les dépenses d'équipement}

Les dépenses d'équipement représentent $128 \mathrm{MF}$ (dont $46 \mathrm{MF}$ dans le Bas-Rhin), assez ciblées sur quelques secteurs comme le montre le tableau suivant :

\section{Répartition des dépenses d'équipement par secteur d'activité}

\begin{tabular}{lcc}
\hline \multicolumn{1}{c}{ Secteurs } & Codes NAF & Poids \\
\hline Fabrication de machines de bureau et matériels info. & 30 & $25 \%$ \\
Fabrication d'appareils et d'équipements élect. & 31 et 33 & $41 \%$ \\
Industrie automobile & 34 & $1 \%$ \\
Industries diverses & 36 & $10 \%$ \\
Construction (5) & 45 & $24 \%$ \\
\hline Total (128 MF) & & $100 \%$ \\
& & $(1996)$ \\
\hline
\end{tabular}

Une première analyse de la répartition du budget d'investissement du pôle universitaire de Strasbourg avec localisation géographique de ses fournisseurs, permet de montrer que seuls $36 \%$ des montants investis se sont portés vers des entreprises du Bas-Rhin. On observe, en outre, que «l'évasion» territoriale est très variable selon les secteurs d'activités concernés puisque pour la construction $83 \%$ des dépenses restent dans l'économie locale contre $2 \%$ pour les achats d'appareils et d'équipements électriques.

Part des dépenses d'équipement réalisées dans le Bas-Rhin, par secteur d'activité

\begin{tabular}{lcc}
\hline Secteurs & Codes NAF & Poids de la dépense dans le Bas-Rhin \\
\hline Fabrication de machines de bureau et matériels info. & 30 & $40 \%$ \\
Fabrication d'appareils et d'équipements élect. & 31 et 33 & $2 \%$ \\
Industrie automobile & 34 & $100 \%$ \\
Industries diverses & 36 & $51 \%$ \\
Construction & 45 & $83 \%$ \\
\hline Total (128 MF) & & $36 \%$
\end{tabular}




\section{b) Les dépenses de fonctionnement}

Les dépenses de fonctionnement ont été analysées selon une méthodologie identique. Elles se montent à $378 \mathrm{MF}$ dont $219 \mathrm{MF}$ dans le Bas-Rhin. Il est à noter cependant que les dépenses en salaires sur budgets propres (174 MF) n'ont pas été intégrées à ce stade de l'analyse puisqu'elles s'inséraient de façon plus cohérente dans la masse salariale globale, c'est à dire pour l'étude de l'impact des consommations du personnel des universités et laboratoires de recherche.

En termes d'impact sectoriel, les consommations des universités ont porté pour l'essentiel sur les postes suivants :

- Achats de petit matériel et de fournitures (38\% des montants analysés). Ces dépenses concernent pour moitié du petit équipement (scientifique, informatique, etc.), et pour un tiers la branche édition, reprographie et papier.

- Prestations de services (environ un quart des consommations des universités). Il s'agit pour moitié de prestations "externes" liées essentiellement aux déplacements des universitaires (frais d'hôtellerie et de transport), et de prestations inhérentes au fonctionnement des universités comme le gardiennage, la maintenance informatique ou le blanchissage.

- Règlement des charges courantes (22\% des budgets de fonctionnement analysés). Ces charges correspondent d'une part aux factures d'électricité, eau, chauffage..., et d'autre part aux frais de poste et télécommunication.

- Dépenses d'entretien du bâtiment (14\% des budgets). Ces dépenses sont relatives essentiellement à des travaux d'isolation, de climatisation et d'entretien des réseaux.

Cette analyse sectorielle nous a conduit à détailler les différents postes de dépenses selon la nomenclature NAF, en observant la localisation des fournisseurs. Les principaux résultats sont les suivants :

\begin{tabular}{lccc}
\hline Secteurs & Code NAF & $\begin{array}{c}\text { Poids dans la } \\
\text { dépense totale }\end{array}$ & $\begin{array}{c}\text { dont part dépensée dans le } \\
\text { Bas-Rhin }\end{array}$ \\
\hline Industrie de l'habillement & 18 & $1 \%$ & $100 \%$ \\
Industrie papier et carton & 21 & $2 \%$ & $60 \%$ \\
Édition, imprimerie, reproduction & 22 & $10 \%$ & $32 \%$ \\
Industrie chimique & 24 & $8 \%$ & $61 \%$ \\
Construction & 45 & $14 \%$ & $64 \%$ \\
Machines de bureau et matériel informatique & 30 & $5 \%$ & $58 \%$ \\
Équipements, appareils électriques & $31,32,33$ & $11 \%$ & $56 \%$ \\
Industries diverses & 36 & $2 \%$ & $60 \%$ \\
Fluides (eau, gaz, électricité) & $23,40,41$ & $15 \%$ & $80 \%$ \\
Commerce et réparation automobile & 50 & $0 \%$ & $47 \%$ \\
Hôtellerie et restauration & 55 & $6 \%$ & $72 \%$ \\
Transports & 60,62 & $8 \%$ & $61 \%$ \\
Postes et télécommunications & 64 & $7 \%$ & $36 \%$ \\
Services informatiques & 72 & $2 \%$ & $27 \%$ \\
Services aux entreprises & 74 & $9 \%$ & $50 \%$ \\
Services personnels & 93 & $0 \%$ & $100 \%$ \\
\hline Total (378 MF) & & $100 \%$ & $58 \%$ \\
& & & $(1996)$ \\
\hline
\end{tabular}

Ces résultats montrent que $58 \%$ des dépenses de fonctionnement (hors salaires) restent dans l'économie locale, ce qui représente un montant de près de $219 \mathrm{MF}$. 
De manière plus agrégée, on obtient :

\begin{tabular}{|c|c|c|}
\hline Postes & $\%$ de la dépense totale & $\%$ de la dépense dans le Bas-Rhin \\
\hline Petits matériels et fournitures & $38 \%$ & $35 \%$ \\
\hline Prestations de service & $26 \%$ & $25 \%$ \\
\hline Charges courantes & $22 \%$ & $25 \%$ \\
\hline Autres & $14 \%$ & $16 \%$ \\
\hline Total (378 MF) & $100 \%$ & $100 \%$ \\
\hline
\end{tabular}

Ces résultats appellent quelques remarques.

- Les achats de petit matériel et de fournitures, premier poste de dépense de fonctionnement des universités (38\%) sont également le premier poste sur la zone cible (Bas-Rhin) mais de manière moins marquée $(35 \%)$ car ces biens matériels sont moins systématiquement achetés localement que les prestations de service ou surtout les charges courantes.

- Le cas des frais de fonctionnement courants (type énergie, poste,...) est particulier : l'existence d'un opérateur régional pour l'électricité (Électricité de Strasbourg) explique la proportion assez élevée de dépenses effectuées dans le Bas-Rhin (80\%), alors qu'on aurait pu s'attendre à un impact local faible.

L'étude des dépenses d'investissement et de fonctionnement montre donc que les fournisseurs locaux restent majoritairement des interlocuteurs commerciaux privilégiés pour le pôle universitaire de Strasbourg, avec cependant une certaine disparité selon les secteurs concernés. Cette partie de l'étude nous a permis d'explorer une dimension intéressante sur le plan méthodologique, avec une analyse détaillée des comptes des établissements, débouchant sur un impact sectoriel précis auprès des entreprises locales. Cependant, nous nous sommes vite aperçus que cet impact des consommations intermédiaires universitaires, même dans le cas d'une grande université à dominante scientifique, reste marginal aussi bien pour l'économie du territoire $(0,2 \%$ du PIB départemental) que pour l'impact global mesuré (9\% de l'impact monétaire direct). Comme nous allons le voir, la majeure partie de l'impact économique direct est en fait lié à l'existence d'une population d'étudiants et de salariés universitaires fixés en région. Traduit en termes d'emplois (cf. partie IV), l'impact des consommations intermédiaires se limite à 300 « équivalents-emplois » dans l'économie locale, chiffre à rapprocher des 5000 salariés directs des établissements. 


\section{L'UNIVERSITÉ : EMPLOYEUR ET LOCALISATEUR D'UNE POPULATION IMPORTANTE}

Le personnel employé par l'ensemble des universités représente un impact économique significatif si l'on s'attache à étudier les dépenses potentielles effectuées sur le territoire du Bas-Rhin (localisation à 90\%). Il en est de même avec les dépenses des étudiants, avec des profils de consommation différents. Ces dernières se sont même révélées très importantes après enquête par sondage.

\section{A - L'impact des consommations du personnel du pôle universitaire}

En 1996, les trois universités de Strasbourg ainsi que les laboratoires du CNRS et de l'INSERM ont employé 4744 salariés pour un budget ministériel d'environ 1,3 MdF. A ce montant s'ajoutent environ $174 \mathrm{MF}$ (budgets propres) versés pour le paiement d'heures complémentaires, de vacations diverses ou d'honoraires. Cette première dépense correspond environ à 880 personnes. Le montant global estimé se monte finalement à près de $1,5 \mathrm{MdF}$.

Cependant, il est à noter que cette somme ne correspond pas à un revenu net global directement consommable. Il faut en effet soustraire les cotisations patronales et sociales, ainsi que l'impôt sur le revenu. Au final, nous pouvons estimer qu'environ $990 \mathrm{MF}$ restent disponibles. L'intérêt de l'étude étant d'évaluer un impact économique et si possible de le quantifier selon les secteurs d'activité, il a été nécessaire de calquer la méthodologie utilisée pour l'analyse des dépenses des universités, c'est-à-dire opérer une conversion dans une table sectorielle telle que la nomenclature NAF.

\section{B - Les difficultés méthodologiques}

L'évaluation de l'impact de cette dépense de 990 MF sur l'économie du Bas-Rhin aurait mérité un traitement analogue à celui qui a été appliqué précédemment aux dépenses de fonctionnement et d'investissement des universités, à savoir une double ventilation par secteurs d'activité et par lieu de consommation.

Cette double analyse n'a malheureusement pu être conduite, faute de sources et de statistiques adéquates. Nous avons été notamment confrontés à deux difficultés :

- En premier lieu, la ventilation des dépenses du personnel universitaire en fonction de son lieu géographique de résidence exact aurait nécessité un travail très lourd avec les services administratifs de chacun des établissements. Cette ventilation n'a donc pas été réalisée.

- En second lieu, la ventilation des dépenses par nature ne repose pas sur une nomenclature d'activités comparable à celle utilisée pour le reste des dépenses des universités. L'impact économique n'a donc pas pu être calculé par branche, mais seulement à un niveau global.

Compte tenu de ces contraintes, nos résultats, fondés sur une répartition des dépenses du personnel universitaire comparable à la structure de consommation des ménages, se présentent par type de consommation et non par secteur d'activité.

\section{C - La structure de consommations des ménages}

L'INSEE fournissant des données précises sur la structure de consommation régionalisée des ménages (6) de la région Est, il a été possible d'estimer les consommations du personnel des universités en correspondance avec le montant global des salaires disponibles versés (990 MF). 


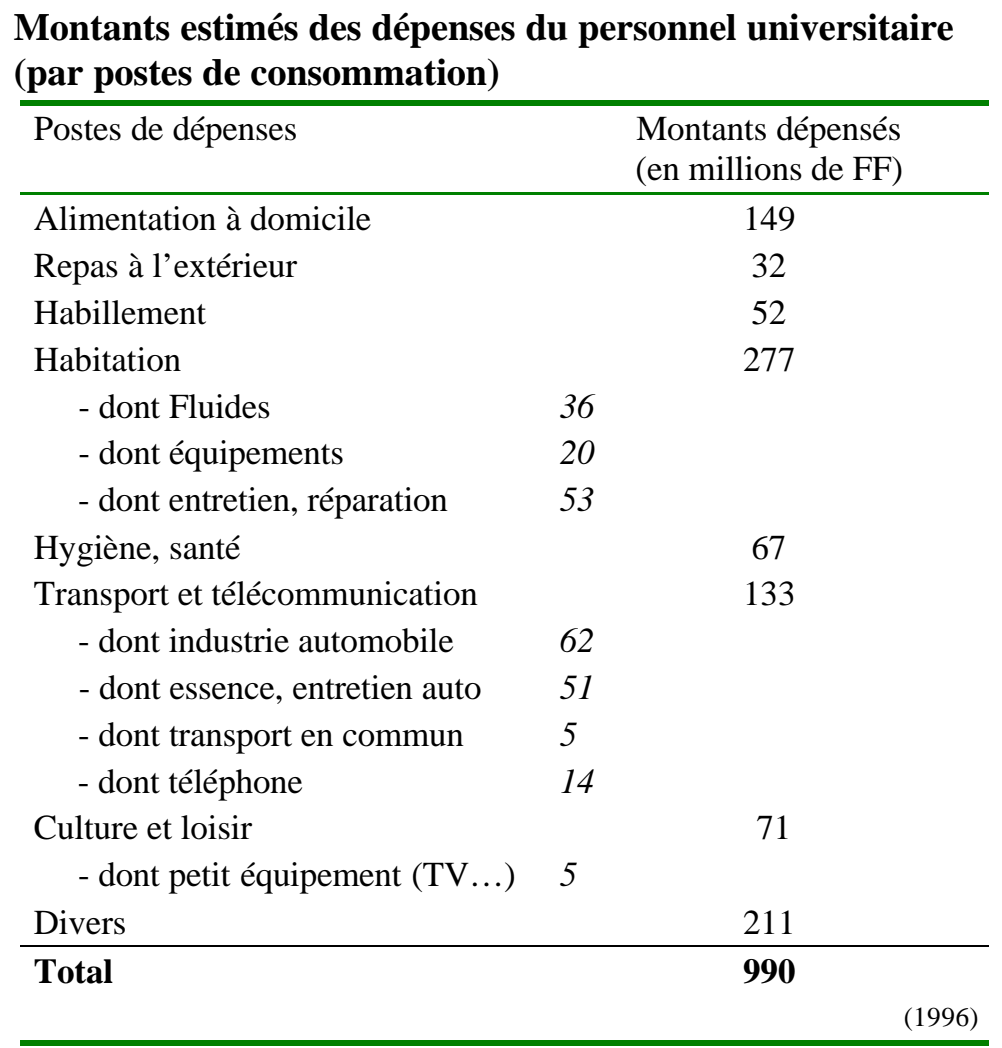

\section{D - L'attraction de la population étudiante}

Le pôle universitaire de Strasbourg regroupe trois universités de grande taille puisque près de 39000 étudiants y faisaient leurs études supérieures en 1996.

Chaque étudiant dispose d'un revenu annuel d'environ $47400 \mathrm{~F}$ selon une estimation obtenue par une enquête spécifique (7). La dépense globale correspondante se monte alors à $1850 \mathrm{MF}$ dont $90 \%$ sont dépensés dans le Bas-Rhin (soit $1700 \mathrm{MF}$ ).

L'enquête spécifique a été menée au cours du premier semestre 1998 sur 450 étudiants représentatifs des trois universités strasbourgeoises, dans l'optique de connaître entre autres leur budget moyen en volume et en structure. Les étudiants interrogés forment un échantillon respectant la répartition par sexe, discipline et niveau dans le cycle d'étude. Le budget mensuel moyen par étudiant approche $4400 \mathrm{~F}$. Il s'agit bien entendu d'une déclaration et non d'une mesure et on a quelques raisons de penser que ce budget est sous-évalué, en particulier pour les étudiants vivant chez leurs parents. On rapprochera ce chiffre de celui estimé par l'Observatoire de la Vie Étudiante en 1997 : 5000 F/mois. Les différences apparaissent aussi, moins nettement, selon le niveau des études : d'environ $4000 \mathrm{~F}$ pour des étudiants de premier cycle, à plus de $5000 \mathrm{~F}$ pour les étudiants de troisième cycle. 


\section{ESTIMATIONS DE L'IMPACT DIRECT EN TERMES D'EMPLOIS}

La mesure de l'impact direct des dépenses des établissements d'enseignement et de recherche, de son personnel salarié et des étudiants attirés sur territoire local va suivre des méthodologies sensiblement différentes selon les cas.

\section{A - La mesure de l'impact sur l'emploi des dépenses de fonctionnement et d'investissement}

Dans le cas présent, il est apparu intéressant d'effectuer des rapprochements entre les dépenses réalisées par les universités et les chiffres d'affaires globaux des secteurs concernés en Alsace (8). Sur cette base ont pu être réalisées des estimations en termes d'emplois, dont les principaux résultats sont les suivants :

- Les achats des universités strasbourgeoises dans 5 secteurs d'activité qui représentent environ $30 \%$ de leur budget de fonctionnement hors salaires, auraient contribué à la création ou au maintien de $\mathbf{4 6}$ emplois, à comparer à des effectifs globaux de plus de 55000 salariés dans ces secteurs (9).

- Étendu à l'ensemble des secteurs productifs ayant été fournisseurs des universités, cet impact en emploi serait sur le Bas-Rhin de l'ordre de 300 emplois.

- En extrapolant ces résultats sur l'ensemble des achats effectués par les universités strasbourgeoises, et non plus seulement sur leur part consommée dans le département, les créations ou maintien d'emplois seraient au total de l'ordre de 350 emplois sur l'ensemble du territoire national.

Ces résultats appellent une double série de remarques, venant à en relativiser la portée :

- En premier lieu, les chiffres avancés sont des estimations rudimentaires. En effet, le caractère très parcellaire de l'information disponible nous a conduit à appliquer un simple coefficient linéaire. Elles donnent néanmoins des ordres de grandeur fiables. Sur la base des calculs effectués, les universités strasbourgeoises contribueraient pour moins de $1 \%$ aux effectifs salariés des branches analysées !

- En second lieu, il est clair que ces ordres de grandeur ne sont pas à l'échelle des véritables enjeux de développement économique que peut apporter une structure universitaire du type de celle de Strasbourg. Le principal impact économique à court terme reste l'emploi direct généré par l'université (près de 5000 emplois). L'autre grand impact (à court terme mais aussi dans une perspective plus dynamique et donc plus difficile à chiffrer) est relatif aux relations avec les entreprises.

Ces résultats appellent aussi une remarque complémentaire d'ordre méthodologique : contrairement à la plupart des analyses d'impact économique d'infrastructures, il a été jugé préférable de ne pas appliquer de coefficient multiplicateur aux résultats bruts, dans la mesure où l'on ne dispose pas d'un modèle économique régional général susceptible de produire un tel multiplicateur, à la différence du niveau national.

De même, les dépenses d'équipement n'ont pas fait l'objet d'une traduction en termes d'emplois, le rythme d'investissement des universités n'étant pas nécessairement régulier. Au total, les chiffres obtenus sont sous-estimés mais fiables (au sens d'une borne inférieure certaine). 


\section{B - Les dépenses de consommation du personnel universitaire et des étudiants}

L'impact économique des universités est, comme indiqué ci-dessus, bien plus important quand on analyse l'effet résultant de la masse salariale universitaire et des dépenses de la population étudiante fixée sur le territoire une grande partie de l'année.

Compte tenu des difficultés méthodologiques mentionnées précédemment, il n'a pas été possible de traduire l'impact de ces dépenses par secteur, et par conséquent de consolider ces résultats avec ceux relatifs aux consommations des universités.

Le rappel de quelques éléments de cadrage permet cependant de mettre en perspective les résultats obtenus avec des données macro-économiques sectorielles régionales :

- Avec leurs 4744 salariés, les universités strasbourgeoises emploient $\mathbf{0 , 7 \%}$ de la population active du Bas-Rhin (hors demandeurs d'emploi). Ce dernier résultat peut, dans une certaine mesure, être réévalué si l'on ajoute le personnel travaillant au sein des universités et payé sur budget propre (dont l'effectif est connu avec moins précision).

- En appliquant le ratio régional alsacien PIB/emploi (10) aux 990 MF de revenus, nets de cotisations et d'impôts, perçus par le personnel universitaire strasbourgeois, on peut estimer à 2759 le nombre d'emplois liés indirectement à la présence des universités et des organismes de recherche strasbourgeois (11).

Au résultat précédent, il faut ajouter les emplois induits par les dépenses des étudiants. On se fondera sur 39000 étudiants et un budget moyen annuel de $47400 \mathrm{~F}$ par personne dont $90 \%$ dépensé dans le Bas-Rhin, d'où un impact de l'ordre de 4647 emplois en utilisant le ratio de 1 emploi pour $358000 \mathrm{~F}$ de revenu dépensé.

Notons que ce résultat diffère sensiblement de celui retenu par Baslé - Le Boulch (1999). En effet, selon eux et sur la base d'un travail effectué par Orivel (1991), la dépense d'un étudiant serait d'environ 2900 F mensuel contre 4400 F de budget estimé sur le site de Strasbourg dans le cadre de notre étude. Hormis cette différence, la présence des étudiants apparait malgré tout comme le moteur économique prépondérant.

Pour être plus précis, il convient de se demander combien de ces emplois sont des emplois supplémentaires par rapport à une situation où il n'y aurait pas d'université. De ce point de vue, on peut supposer qu'une petite partie des étudiants concernés serait présente sur le territoire alsacien.

On peut donc corriger à la baisse l'impact des dépenses des étudiants. Cette démarche a été rendue possible pour l'étude menée sur l'université de Montréal par l'existence d'un dispositif statistique régional dont on ne dispose pas pour les régions françaises. L'argument, souvent mis en avant, de la forte contribution des étudiants à l'économie locale demande donc à être pris avec prudence.

Cette réduction de l'impact de la dépense étudiante ne peut être estimée que de manière grossière, et nous conduit à ramener le nombre d'emplois induits de 4647 à environ 3000.

Au total, l'impact des dépenses du personnel universitaire et des dépenses des étudiants est de l'ordre de 6000 emplois, auxquels s'ajoutent les 5000 emplois directs au sein des universités.

À côté de ces 11000 emplois (directs et générés par les revenus des étudiants et personnels), les quelques 300 emplois supplémentaires générés par les consommations intermédiaires étudiés en (A) ci-dessus pèsent peu. 


\section{L'IMPACT INDIRECT SUR L'ÉCONOMIE LOCALE}

L'impact d'un pôle universitaire reste donc relativement modeste si on le considère comme un agent économique ordinaire (consommateur direct). C'est par rapport à sa vocation première de centre d'enseignement supérieur et de recherche que son influence se fait vraiment sentir. En effet, une université va jouer un rôle prépondérant dans le domaine de la qualification de la main d' auvre locale, dans le domaine de la recherche et du développement industriel (y compris comme générateur de développement endogène via l'essaimage) et comme pôle d'attraction pour des entreprises souhaitant bénéficier de cet environnement.

Cet impact apparaît dans toute son ampleur si l'on considère que les universités de Strasbourg et leurs laboratoires ont su acquérir une renommée internationale et que leur implantation s'insère dans la patrimoine historique de l'Alsace (12). La caractéristique particulièrement fondamentale de la recherche strasbourgeoise n'est pas forcément un obstacle à son impact économique, si l'on suit le raisonnement de Keith Pavitt (1993) selon lequel l'entreprise tire profit du potentiel de recherche fondamentale sous des formes qui ne sont pas nécessairement la production de savoir codifié, mais plutôt les compétences en résolution de problèmes (ces compétences sont plus appropriables localement que les productions scientifiques elles-mêmes).

Pour mener à bien cette approche, notre objectif a été essentiellement de préciser la relation que peuvent entretenir les universités avec les entreprises régionales. Sur ce plan, nos préoccupations portaient essentiellement sur :

- la connaissance et l'appréciation de l'offre universitaire en formation et en recherche,

- le poids des universités dans la décision d'implantation des entreprises,

- les divers modes de relation entreprise-université et leurs perspectives de développement.

\section{A - Les principes méthodologiques}

Cette étude s'est déroulée en deux phases successives.

La première étape a consisté à définir un panel représentatif de 1200 établissements d'entreprises (13) du Bas-Rhin auprès desquels ont été effectués 400 entretiens téléphoniques. Le choix de l'échantillon répondait à une double préoccupation méthodologique :

- d'une part, retenir le plus de secteurs d'activité possible, même si les liens avec les universités paraissent pour certains faibles ou nuls ;

- d'autre part, prendre un nombre plus grand d'entreprises implantées dans la Communauté Urbaine de Strasbourg (CUS), aire d'influence "naturelle" de l'ensemble universitaire strasbourgeois.

Les principales caractéristiques des 400 établissements d'entreprises enquêtées sont les suivantes :

- $75 \%$ sont situées sur la CUS et $25 \%$ sur le reste du Bas-Rhin ;

- 4 établissements d'entreprises sur 5 sont implantés en Alsace depuis plus de 10 ans ;

- $70 \%$ comptent de 10 à 50 salariés, et $30 \%$ ont plus de 50 salariés ;

- enfin, ces établissements d'entreprises relèvent à $44 \%$ des services, $38 \%$ de l'industrie, $12 \%$ de la construction et $6 \%$ du commerce.

L'enquête générale a été complétée par une étude plus qualitative menée auprès d'une vingtaine d'entreprises, spécialement sélectionnées pour leur proximité avec le milieu universitaire. Il s'agissait de réaliser un entretien ouvert approfondi avec certains des dirigeants locaux afin d'évaluer plus précisément les pratiques de recrutement, les stratégies économiques et le degré d'adaptation de l'offre universitaire à leurs besoins de compétences. Il est évident que l'interprétation des résultats tirés de ce 
dernier travail doit être considéré avec prudence et n'a pas valeur de généralité. Ce travail s'avère cependant riche d'enseignements et complète utilement les conclusions de l'enquête téléphonique.

\section{B - Les principaux résultats}

L'appréciation spontanée des entreprises est globalement bonne, avec cependant des différences sensibles selon les thèmes :

- La qualité des enseignements est très largement reconnue.

- La question de l'adaptation des enseignements aux besoins suscite à la fois le nombre le plus élevé de réponses (74\% d'opinions exprimées) et de réponses négatives (l'adaptation est jugée mauvaise à $21 \%$ ).

- La question du dynamisme de la recherche est, elle, dans un cas de figure symétrique, avec peu d'opinions exprimées (40\%) mais un très fort taux de satisfaction (71\%).

Concernant le degré de connaissance de l'offre universitaire en formation et en recherche, $48 \%$ seulement des entreprises interrogées déclarent connaître bien ou moyennement les cursus de formation proposés, et $30 \%$ les thèmes de recherche des différents laboratoires. Ce résultat est à pondérer en fonction de la taille de l'entreprise et du secteur d'activité puisque ce sont surtout dans le domaine des services et pour les entreprises de plus de 50 salariés que les cursus et la recherche du pôle universitaire de Strasbourg sont les mieux cernés. Le constat global reste que la connaissance spontanée que les entreprises ont des universités est décevant (e qui suggère de mener une véritable politique de communication).

Un autre thème, a priori fondamental dans une tentative d'examen de la relation UniversitéEntreprise est le poids que représente la présence académique dans une décision d'implantation locale. En effet, pour des raisons de dynamisme économique, de compétence de la main d' ouvre, de proximité de stagiaires, de partenariat en recherche et développement ou simplement pour l'opportunité d'offrir à son personnel des facilités d'études supérieures pour leurs enfants, l'existence d'un grand pôle universitaire doit jouer un rôle significatif dans la décision finale.

Interrogées à ce propos, $10 \%$ des entreprises ont considéré les universités comme un facteur de premier ou second rang dans la décision d'implantation (14). Les $90 \%$ d'entreprises restantes confirment le constat global que l'on peut tirer de cette enquête : un manque certain de communication entre les universités et le monde de l'entreprise ordinaire.

Enfin, les liens qui unissent les entreprises et le milieu académique sont essentiellement de quatre types :

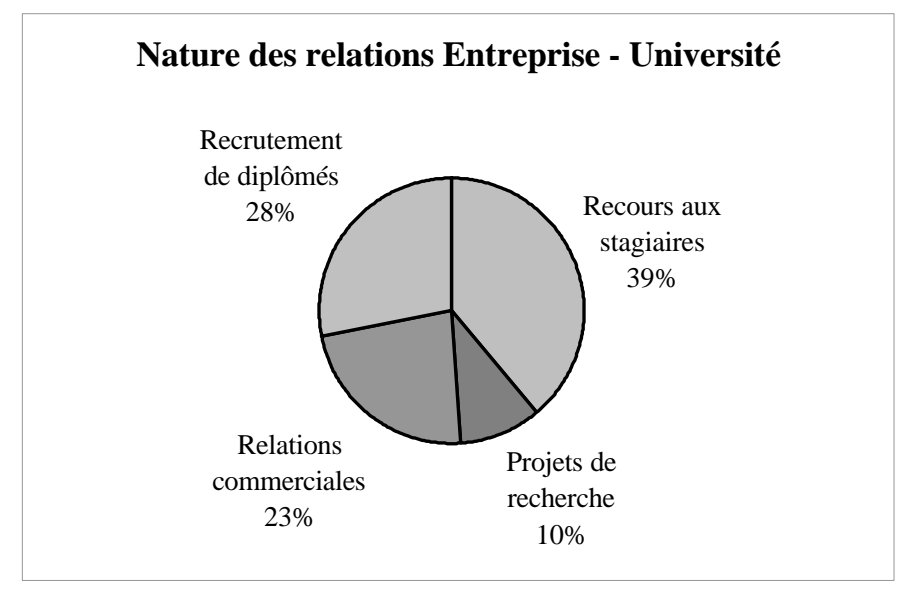

L'enquête montre que $35 \%$ des entreprises de l'échantillon n'ont aucun rapport avec le monde académique. Sur les relations déclarées, on notera l'importance du recrutement des stagiaires. Quant 
aux cas de relations de recherche, quelle que soit l'importance de ce type d'impact à long terme sur le développement territorial, il reste le fait d'une catégorie très spécifique d'entreprises.

La question du recours à des personnes formées à l'université, pour des stages ou pour des embauches, mérite quelques approfondissements puisque c'est par ce biais que l'ancrage territorial des universités semble le plus important. De ce point de vue, l'enquête apporte un éclairage intéressant sur les liens entre, d'une part, recrutement et connaissance de l'offre de formation, et d'autre part, recrutement et stage.

- Les entreprises qui recrutent des étudiants de l'université connaissent l'offre de formation qui y est proposée. A l'inverse, sur les 253 entreprises qui déclarent ne jamais embaucher de diplômés de l'université, 160 (soit les deux tiers) connaissent mal ou pas du tout cette offre.

- Sur les 185 entreprises employant régulièrement ou occasionnellement des stagiaires, 110 (soit $60 \%$ d'entre elles) embauchent des diplômés de l'université.

Soulignons tout particulièrement que, malgré des relations encore trop lointaines entre les grandes entreprises et le monde universitaire, l'enquête met en évidence une volonté certaine de développer la connaissance réciproque.

\section{C - Analyse de la relation contractuelle}

Les entretiens en entreprise n'ont pas permis de mettre en évidence une coopération forte en termes de recherche coopérative des universités avec leur environnement économique proche, même si ce constat reste à relativiser, compte tenu de la taille très réduite (et sans prétention de représentativité) de l'échantillon d'entreprises contactées, pour ces entretiens approfondis. Nous savons par les sources officielles qu'une bonne partie des relations qu'entretiennent les universités strasbourgeoises avec leur environnement, en particulier les entreprises, passe par l'élaboration d'études, de contrats et de missions de recherche et d'analyses diverses, qui représentent plus de 70 millions de $\mathrm{F}$ pour l'ensemble des universités de Strasbourg et de ses laboratoires associés (60\% de contrats publics et $40 \%$ de contrats avec des organisations privées).

En matière d'études sur appels d'offre publics, seuls $17 \%$ sont destinées à la région Alsace. Quant aux contrats industriels, la mesure précise de leur localisation géographique n'a pas été réalisée dans le cadre de l'étude dont nous rendons compte ici. Il n'est d'ailleurs pas très facile d'identifier les entreprises alsaciennes prenant part aux contrats. De plus cette question n'est pas essentielle car, bien souvent, ce n'est pas avec «l'unité alsacienne » que l'université contracte mais directement avec le siège, lequel est fréquemment dans le reste de la France ou à l'étranger pour les grandes entreprises partenaires de la recherche universitaire. Ce qui importerait plus c'est de savoir si le résultat de ces contrats de recherche a un impact sur l'activité en Alsace de l'entreprise. Mais cela relèverait d'une analyse beaucoup plus détaillée de ces contrats sur une longue période. 


\section{CONCLUSION}

L'analyse d'impact des Universités de Strasbourg (et des organismes de recherche associés) nous a permis de tirer plusieurs enseignements sur le rôle économique local d'un grand pôle d'enseignement et de recherche. Mais tout d'abord, sur le plan méthodologique (cf. le tableau de synthèse ci-dessous), sont apparues des difficultés de collecte et d'interprétation des sources comptables que nous n'attendions pas. On doit pouvoir améliorer cet aspect du travail, mais certainement au prix d'un effort important et ingrat, qui suppose aussi de solliciter fortement les bonnes volontés des personnels administratifs des établissements, voire de dépasser certains cloisonnements entre les différents organismes.

En ce qui concerne le fond, il apparaît tout d'abord que les effets des dépenses du personnel et surtout des étudiants attirés sur le site forment l'essentiel de l'impact économique immédiat. On observe ensuite des répercussions directes sur le monde de l'entreprise via l'amélioration du capital humain mais aussi selon des modalités assez particulières révélées par l'enquête (rôle essentiel d'un vivier de stagiaires potentiels par exemple). Nous montrons également que la relation université-entreprise reste plus distante encore que l'on pouvait le supposer initialement à cause, en partie, de la mauvaise lisibilité des cursus universitaires de la part des entrepreneurs. Mais il s'agit là d'une vision moyenne qui recouvre une forte diversité : à côté d'une majorité d'entreprises plutôt éloignées du milieu académique, on trouve une minorité (de l'ordre de $10 \%$ ) très motivée.

La grande question, non traitée dans cette étude et difficile à aborder, est celle de l'impact à long terme sur les trajectoires d'évolution du milieu économique local. En particulier on peut se demander si la minorité d'entreprises fortement impliquées avec l'université n'est pas, en fait, typiquement représentative du tissu industriel à venir, dans le cadre de la nouvelle «économie fondée sur la connaissance ».

L'évaluation à laquelle nous avons procédé était principalement axée sur les effets directs. Elle reste à compléter non seulement par une enquête plus large auprès des partenaires comme les entreprises, mais aussi sur les activités diverses générées par le monde de la recherche : organisation de colloques et autres activités annexes; effet de levier dans le domaine des infrastructures, des équipements et de l'image du territoire ; création d'entreprises fondées sur la science, etc.

L'influence à long terme du système académique sur le développement régional (et en particulier urbain) est un fait majeur qui n'est pas analysé ici, puisque notre objectif était d'évaluer un impact immédiat. Ce dernier est un bon exemple du rôle joué par le financement largement exogène (en France sur le budget de l'État) du système académique dans l'économie locale. Mais le monde universitaire est, à long terme, une importante source de développement endogène, par exemple via la création de nouvelles activités, comme de développement exogène en participant fortement à l'attractivité du territoire. 
Synthèse méthodologique de l'étude

\begin{tabular}{|c|c|}
\hline \multicolumn{2}{|c|}{ Mesure de l'impact direct } \\
\hline Méthodologie & Difficultés rencontrées \\
\hline $\begin{array}{l}\text { Fonction de consommation } \\
\text { - Analyse détaillée des comptabilités des structures } \\
\text { d'enseignement et de recherche (distinction entre } \\
\text { dépenses de fonctionnement et d'investissement, } \\
\text { agrégation des postes de dépenses et traduction } \\
\text { selon la nomenclature NAF de l'INSEE). } \\
\text { Localisation et identification des fournisseurs } \\
\text { (analyse et agrégation des montants selon la } \\
\text { nomenclature NAF), puis identification de } \\
\text { l'impact direct des dépenses en fonction des } \\
\text { secteurs d'activités et de la localisation. }\end{array}$ & $\begin{array}{l}\text { - Homogénéité imparfaite des comptabilités des } \\
\text { établissements d'enseignement et de recherche } \\
\text { (agrégation et comparaison des postes comptables } \\
\text { parfois délicates). } \\
\text { Imprécision de certains postes de dépenses ou de } \\
\text { recettes. } \\
\text { Non correspondance des postes comptables avec la } \\
\text { nomenclature NAF (construction nécessaire d'une } \\
\text { table de conversion basée sur les intitulés des } \\
\text { postes comptables ou sur la nature des achats). }\end{array}$ \\
\hline $\begin{array}{l}\text { Fonction de localisation d'une population } \\
\text { - Évaluation précise du montant global des salaires } \\
\text { du personnel des universités. } \\
\text { Les ressources et les dépenses des étudiants ont } \\
\text { fait l'objet d'une enquête sur un échantillon } \\
\text { représentatif de } 450 \text { individus (estimation possible } \\
\text { de la répartition et de la nature des revenus ainsi } \\
\text { que des dépenses moyennes par étudiant). }\end{array}$ & $\begin{array}{l}\text { - Lieu de résidence du personnel universitaire } \\
\text { difficile à préciser (sauf à mener un travail } \\
\text { d'enquête très lourd). } \\
\text { - Nature et répartition des dépenses de ces ménages } \\
\text { en région impossibles à quantifier avec précision } \\
\text { (aussi avons nous utilisé les indices de répartition } \\
\text { de la consommation des ménages calculés par } \\
\text { l'INSEE). } \\
\text { Impossibilité de calculer une répartition des } \\
\text { consommations par branche (les indices précédents } \\
\text { sont par «postes » de consommation). }\end{array}$ \\
\hline $\begin{array}{l}\text { Traduction de l'impact en termes d'emplois } \\
\text { - Application de ratios du PIB régional par branche } \\
\text { et par emploi aux dépenses universitaires pour les } \\
\text { branches considérées. } \\
\text { Application des ratios obtenus à la répartition des } \\
\text { consommations des ménages (personnel des } \\
\text { universités et étudiants). }\end{array}$ & $\begin{array}{l}\text { - Absence de modèle macroéconomique régional } \\
\text { permettant de disposer de multiplicateurs précis. } \\
\text { - En raison du caractère irrégulier des dépenses } \\
\text { d'investissement d'une année sur l'autre, nous } \\
\text { n'avons pas traduit en termes d'emplois les } \\
\text { montants approximatifs de cet impact direct (qui } \\
\text { reste relativement marginal). }\end{array}$ \\
\hline \multicolumn{2}{|c|}{ Mesure de l'impact indirect } \\
\hline $\begin{array}{l}\text { - Définition d'un échantillon représentatif (toutes } \\
\text { branches confondues) de } 1200 \text { entreprises } \\
\text { régionales (envoi d'un questionnaire). } \\
\text { Définition d'un sous-échantillon représentatif de } \\
400 \text { entreprises (respect de la répartition } \\
\text { géographique, de la taille et du secteur d'activité } \\
\text { des entreprises) pour interviews téléphoniques } \\
\text { - Sélection de } 20 \text { entreprises en relation directe avec } \\
\text { les universités pour des entretiens approfondis } \\
\text { Questionnaire portant aussi bien sur la nature } \\
\text { commerciale de la relation entreprises-université } \\
\text { que sur la connaissance du monde universitaire, les } \\
\text { synergies de R.\&D., l'emploi de stagiaires } \\
\text { étudiants et le recrutement de diplômés, etc. }\end{array}$ & $\begin{array}{l}\text { - Faible taux de réponse des entreprises sollicitées } \\
\text { par voie postale. } \\
\text { - Facteurs qualitatifs parfois difficiles à appréhender } \\
\text { comme l'influence de la présence d'un pôle } \\
\text { universitaire sur les décisions d'implantation, etc. } \\
\text { - Une grande part des effets indirects à long terme } \\
\text { n'est pas évaluée (transfert de technologies, effets } \\
\text { d'image, etc.) bien que ces questions aient été } \\
\text { évoquées dans les questionnaires. }\end{array}$ \\
\hline
\end{tabular}




\section{REFERENCES BIBLIOGRAPHIQUES}

BASLÉ M., J.-L. LE BOULCH, 1999, L'impact économique de l'enseignement supérieur et de la recherche publique sur l'agglomération de Rennes, Revue d'Économie Régionale et Urbaine, $\mathrm{n}^{\circ} 1$, pp. 115-134.

BARRÉ R., GIBBONS M., MADDOX J., MARTIN B., PAPON P., 1997, Science in Tomorrow's Europe, Economica International.

BETA, 1993, Impact de l'Université Louis Pasteur sur son environnement économique et social, en particulier en région, Université Louis Pasteur, Strasbourg I.

BURETH, A., HERAUD, J-A. 2001, "Institutions of technological infrastructure (ITI) and the generation and diffusion of knowledge" in K. Koschatzky, M. Kulicke, A. Zenker (eds.) Innovation networks. Concepts and challenges in the European perspective, Physica Verlag, Springer (69-91).

CAHIER DE L'OBSERVATOIRE DE LA VIE ÉTUDIANTE, 1996, Les conditions de vie des étudiants, La Documentation Française, Paris.

DATAR 2000, "Schéma de services collectifs de l'enseignement supérieur et de la recherche" (document soumis à la consultation), Ministère de l'Éducation Nationale et Ministère de la Recherche, Automne.

GAUDEMAR J.-P., 1991, Formation et développement régional en Europe, DATAR .

INSEE, Tableaux de l'Économie Alsacienne - 1994/1995, Strasbourg.

INSEE, Le budget des ménages en 1995, Statistiques, Consommation et mode de vie.

KLOSTERMAN, R.E., 1990, Community and Analysis Planning Techniques, Rowmand and Littlefield Pub., Savage, Maryland.

MANSFIELD E., 1995, Academic research underlying industriel innovations : sources, characteristics, and financing, Review of Economics and Statistics, 77 (1), February, pp. 55-65.

MARTIN F., 1996, Retombées économiques des activités de recherche de l'Université de Montréal et des écoles, hôpitaux et instituts affiliés - Université de Montréal / Département de sciences économiques.

MARTIN F., 1997, The economic impact of Canadian University R\&D, Research Policy, 27 (7), november, pp. 677-687.

MUSTAR P., 1999, Tableau de bord de la R\&D en Alsace, La Recherche, $\mathrm{n}^{\circ} 325$, novembre, pp. 4445.

OBSERVATOIRE DES SCIENCES ET TECHNIQUES (OST), 1998, Science et technologie Indicateurs, Economica.

ORIVEL F., 1991, L'université, une bonne affaire pour les communes, Le Monde, 07 février.

PAVITT K., 1993, What do firms learn from basic research, in D. FORAY, Ch. FREEMAN, Technology and the Wealth of Nations (eds.), OECD, Pinter Pub., London, pp. 29-40. 


\section{Notes}

(1) Le document qui a fourni la matière à cet article est une étude réalisée par le BETA (UMR 7522 du CNRS et de l'Université Louis Pasteur - Strasbourg I) et BETURE-CONSEIL, pour le compte de la Caisse des Dépôts et Consignations et du Pôle Universitaire Européen de Strasbourg.

(2) Voir par exemple plusieurs des contributions à l'ouvrage collectif de R. Barré et al. (1997), en particulier celle de H. Nowotny, qui parle même d'un «nouveau culte de l'évaluation » et affirme : « the demand for accountability assumes that science has lost much of its previous privileged status and is to be treated in ways similar to other social institutions » (op. cit. p 87).

(3) Compte tenu de la parution récente de cet article, nous ne pouvions tenir compte de l'expérience de Rennes dans la conception de notre propre étude sur Strasbourg (on peut dire que les deux projets ont été approximativement menés en parallèle et sans connaissance mutuelle). Il est d'autant plus intéressant de relever que la méthodologie et les résultats se sont révélés proches à une différence près : ayant adopté une approche plus restrictive de la mesure, nos résultats aboutissent à un impact sous-estimé selon les normes de Baslé, Le boulch (1999). Nos chiffres doivent être compris comme une borne inférieure de l'impact économique.

(4) Aux États-Unis il n'en est pas de même du fait de forts droits d'inscription. Ainsi, l'évaluation de l'Université de Harvard pour l'année universitaire 1997/1998 fait apparaitre 450000 \$ de frais de scolarité sur un revenu total de $1680000 \$$, ce qui permet donc d'évaluer à $27 \%$ la part de l'impact direct lié à la fonction éducative (chiffres obtenus sur le site Internet de l'Université). Ensuite, cet impact peut être régionalisé en observant l'origine géographique des étudiants: par exemple, quelle proportion vient de l'extérieur de l'aire métropolitaine de Boston, ce qui est un gain net pour cette dernière?

Notons que les grandes universités américaines se préoccupent activement de mesurer leur impact économique comme le révèlent leurs sites Internet.

Site Internet de l'Université de Harvard :

http://www.hno.harvard.edu/community/report/

Site Internet de l'université de McGill :

http://www.mcgill.ca/public/releases/1998/january/economy/

Site Internet de l'UCI (University of California, Irvine) :

http://www.communications.uci.edu/eir/eir98.html

Site Internet de Tarleton State University-Stephenville :

http://www.tarleton.edu/main/executivesummary.html

Site Internet de University of Colorado :

http://www.cu.edu/cuimpact/home.html

The Regional Economic Impacts of Idaho State University, 1996 :

http://www.isu.edu/cbr/ISU_Impact_Study/ISU_ImpactStudy8.html

(5) Les dépenses de construction retenues ici sont celles effectuées sur budgets propres des établissements d'enseignement et de recherche et ne correspondent qu'à une faible proportion des montants versés pour les investissements en construction de bâtiments neufs.

(6) « Le budget des ménages en 1995 », INSEE Statistiques, Consommation et mode de vie, 1995. 
(7) L'enquête a été réalisée par des étudiants en Sciences Sociales de l'université Marc Bloch sous la direction de Claude Régnier.

(8) Sources : INSEE, Tableaux de l'Économie Alsacienne, 1994/1995.

(9) L'ensemble de ces cinq secteurs (textile, habillement; papier, carton; imprimerie, édition ; industrie chimique ; transports) génère un chiffre d'affaire de $54011 \mathrm{MF}$ en Alsace pour un effectif total de 55727 personnes. On en peut en conclure d'abord qu'il faut approximativement $1 \mathrm{MF}$ de chiffre d'affaire pour créer un emploi. Par ailleurs, on peut calculer la dépense universitaire dans chacune de ces branches de la manière suivante:

Estimation des créations d'emplois liées à certaines consommations des universités dans le Bas-Rhin

\begin{tabular}{|c|c|c|c|c|c|c|}
\hline Secteurs & $\begin{array}{l}\text { Codes } \\
\text { NAF }\end{array}$ & $\begin{array}{l}\text { CA Alsace } \\
\text { (en MF) }\end{array}$ & \multicolumn{2}{|c|}{$\begin{array}{c}\text { CA lié aux universités } \\
\text { KF } \quad \%\end{array}$} & $\begin{array}{c}\text { Effectif total } \\
\text { du secteur }\end{array}$ & $\begin{array}{l}\text { Impact des } \\
\text { universités }\end{array}$ \\
\hline Textile, & 18 & 9648 & 1691 & $0.018 \%$ & 15142 & 3 \\
\hline habillement & 21 & 7395 & 3357 & $0.045 \%$ & 6882 & 4 \\
\hline Papier, carton & 22 & 3878 & 9396 & $0.242 \%$ & 5897 & 15 \\
\hline Impression, édition & 24 & 9438 & 15442 & $0.164 \%$ & 6106 & 10 \\
\hline $\begin{array}{l}\text { Industrie chimique } \\
\text { Transport }\end{array}$ & $60+62$ & 23652 & 14730 & $0.062 \%$ & 21700 & 14 \\
\hline Total & & 54011 & 44615 & $0.083 \%$ & 55727 & 46 \\
\hline
\end{tabular}

(10) Le calcul «taux d'emploi » (1 emploi généré par $358000 \mathrm{~F}$ de chiffre d'affaires) a été effectué sur la base des données macro-économiques régionales alsaciennes, faute d'informations statistiques pour le département du Bas-Rhin. Pour calculer ce chiffre, qui n'est qu'un ordre de grandeur, nous avons utilisé le PIB et la population active régionale. Ce chiffre de $358000 \mathrm{~F}$ par emploi est plus faible que le ratio de $1 \mathrm{MF}$ par emploi calculé pour les consommations intermédiaires : cela s'explique par la nature moins capitalistique de l'ensemble de l'économie en comparaison des cinq seules branches industrielles concernées par les consommations intermédiaires.

(11) Cette estimation doit être considérée avec précaution car elle repose sur l'hypothèse que l'intégralité du revenu du personnel universitaire strasbourgeois est consommée dans le Bas-Rhin.

(12) L'Empire allemand, de 1870 à la fin de la première guerre mondiale, puis la République française ont successivement cherché à renforcer le rôle de Strasbourg comme vitrine de la science nationale vis-à-vis de l'étranger. Pour des raisons stratégiques, par contre, ni les Allemands ni les Français n'ont implanté en Alsace de grands établissements technologiques (nucléaire, télécom, etc.), ce qui a renforcé la spécialisation «fondamentaliste» du pôle scientifique strasbourgeois. Cette double dimension d'excellence scientifique et de caractère fondamental apparait nettement dans les statistiques scientométriques comme le montre P. Mustar (1999).

(13) Nous avons travaillé sur la base des fichiers de l'INSEE pour les établissements de plus de 20 salariés de la CUS, et des fichiers de la Chambre de Commerce et d'Industrie du Bas-Rhin pour le reste de l'échantillon.

(14) Les principaux facteurs d'implantation sont relatifs à la situation géographique favorable de Strasbourg (37\%), à la proximité du marché (25\%), à l'origine alsacienne des dirigeants (25\%), et à des logiques internes aux groupes auxquels appartiennent les établissements (12\%). Concernant l'approche par l'offre d'éducation pour les enfants des employés, 3\% des entreprises interrogées considèrent qu'il s'agit d'un facteur très important, et $5 \%$ d'un facteur de moyenne importance. 